\title{
Biospecimen Collection Method
}

National Cancer Institute

\section{Source}

National Cancer Institute. Biospecimen Collection Method. NCI Thesaurus. Code C70700.

A method or procedure by which biological material is obtained from a subject for testing, diagnostic, treatment, or research purposes. 